\title{
Soft Microscopy: Multimodal, Correlative and Dynamic Characterization of Soft and Hybrid Structures
}

\author{
Vinayak Dravid
}

Northwestern University, Evanston, Illinois, United States

There has been remarkable progress in broad and diverse areas of materials in recent two decades, since the inception of nanoscale synthesis and architecturing of soft and hard-soft (hybrid) systems. The latter structures, in particular, present daunting challenge for characterization and analysis. This is due to diverse and often diametrically opposite considerations for characterization, especially electron microscopy and analysis. The soft structure necessitates low-dose and/or cryo-mode for microscopy and compels contrast enhancement through staining and related approaches. On the other hand, the hard part of the systems is often robust against radiation abuse in microscopy/analysis. These contraindicating considerations make both soft and hybrid microscopy particularly challenging.

At Northwestern University's NUANCE Center, we have been developing "hardware" as well as methods and approaches for multimodal, correlated and dynamic characterization of soft and hybrid systems - both, under room temperature and cryogenic conditions. The hybrid systems that are being developed are diverse, and encompass classical soft-hard interfaces such as bacterial growth on hard implants or complex polymer-nanoparticle systems; to more exotic ones like DNA-assembled nanoparticles or Covalent/Metal-Organic Framework (COF/MOF) structures and their composites. To mitigate the contrast issues in soft part of the hybrid systems, we invoke the modification of the so-called ChromEMT $^{1}$ approach for enhancing contrast of macromolecular structures. The nominal staining approach is extended to other systems to enhance contrast. In the meanwhile, sparse and dynamic sampling further reduce the deleterious electron flux which otherwise compromises the sample stability. The recent addition of high-performance direct-electron detectors (e.g., so-called K2/K3 cameras) provide additional benefit of sensitivity, thereby helping further reduce electron flux for a given contrast threshold. The concept of "Soft Microscopy" also extends to correlative characterization, typically with optical, confocal/fluorescence or related measurements and in some cases scanned probe microscopy. In some cases, the complementarity is achieved through synchrotron $\mathrm{x}$-ray scattering that provide reciprocal information, which may need real-space modeling through microscopy.

The presentation will cover several examples of this complex choreography of multimodal and correlative characterization of soft and soft-hard hybrid systems, including cryogenic measurements. For example, in case of complex architecture comprising DNA-bound nanoparticles, we show formation of complex structures and phases, revealed by cryo-TEM (Figure 1). In some cases, ultrafine DNAconjugated Au nanoparticles act as "free electrons" in a "nanoparticle crystal" or form localized accumulation akin to the solid-state behavior of metals and insulators ${ }^{2}$. The stability and quality of such data are enhanced with the use of low temperature operation. We show that it is possible to incorporate sampling approach to minimize electron flux for spectroscopy and spectroscopic imaging necessary for EDS and EELS. Figure 2 shows the scheme employed under cryo-TEM to obtain section-by-section stacking info. The availability of direct-electron detectors and accompanied software have transformed imaging and analysis of many other soft and hybrid structures such as COFs and MOFs.

The presentation will cover emerging examples of soft and hybrid materials examined through the multimodal and correlative characterization, including under cryogenic conditions. ${ }^{3}$ 

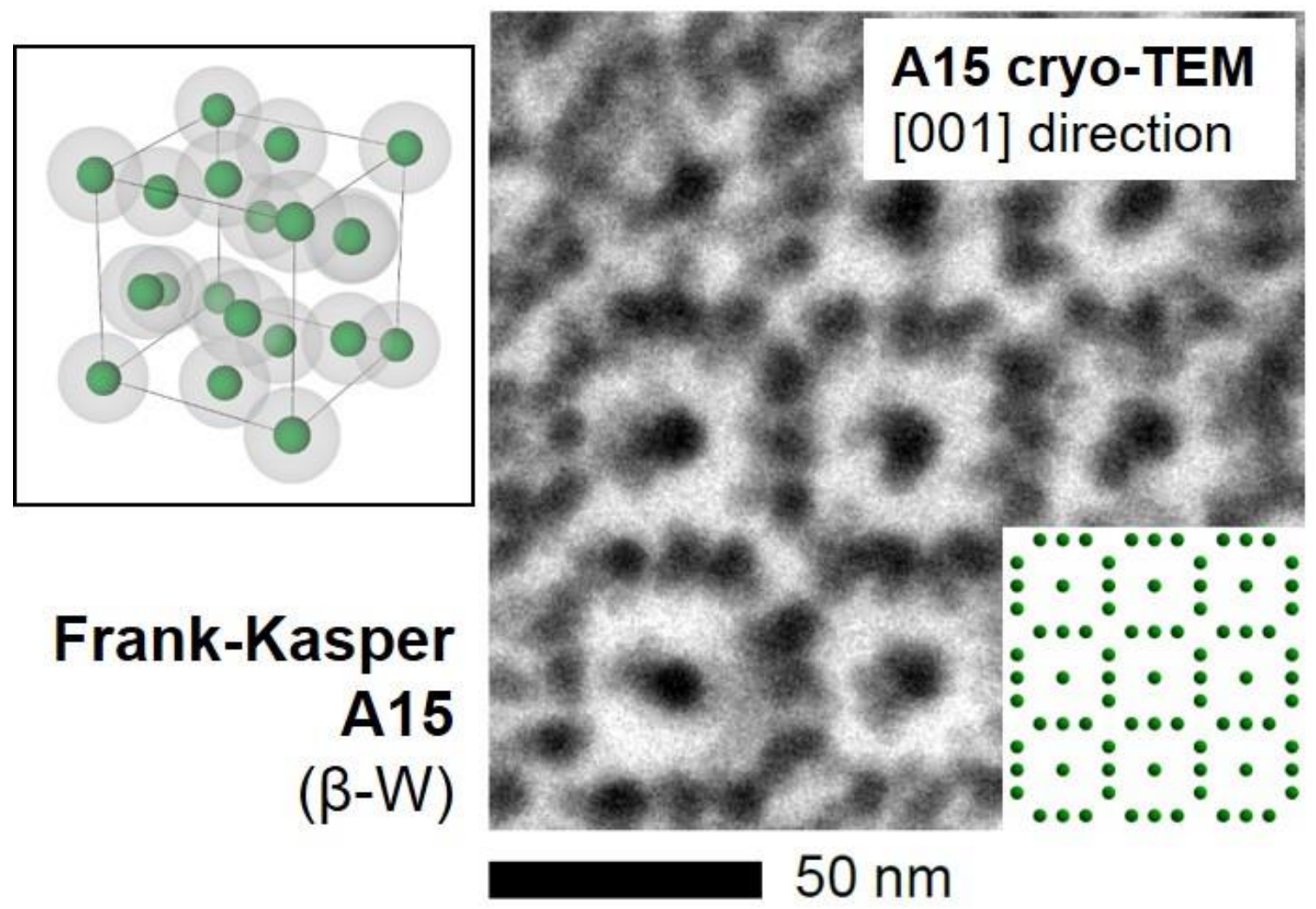

Figure 1. Unusual structures can form during crystallization of nanoparticle-DNA complex. Here is an example of A15 Frank-Casper phase revealed by cryo-TEM.
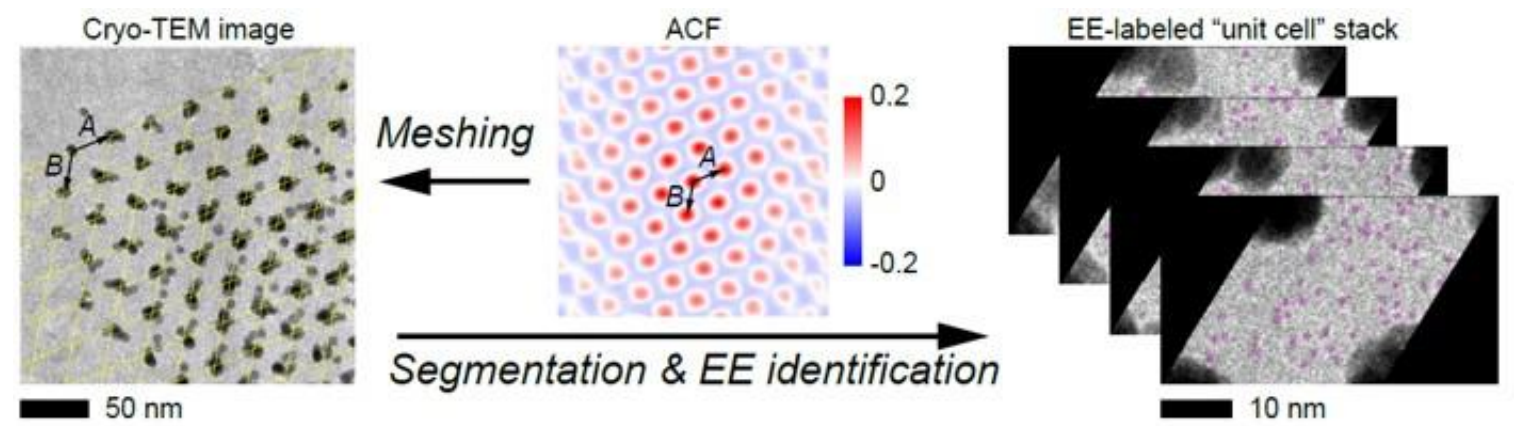

Figure 2. Experimental scheme to extract the nature of "Electron-Equivalent (EE)" nanoparticles from cryo-TEM imaging. (From Ref. 2).

\section{References}

[1] HD Ou, et al, Science (2017), Vol. 357, Issue 6349, eaag0025; DOI: 10.1126/science.aag0025;

[2] M. Girard et al., Science (2019), Vol. 364, Issue 6446, pp. 1174-1178; DOI: 10.1126/science.aaw8237; [3]This material is based on research sponsored by the Air Force Research Laboratory under agreement No. FA8650-15-2-5518, Air Force Office of Scientific Research under Award No. FA9550-12-1-0280. This work made use of the EPIC and BioCryo facilities of Northwestern University's NUANCE Center, which has received support from the Soft and Hybrid Nanotechnology Experimental (SHyNE) Resource (NSF ECCS-1542205); the MRSEC program (NSF DMR-1720139) at the Materials Research Center; the International Institute for Nanotechnology (IIN); the Keck Foundation; and the State of Illinois. 